\title{
Carbohydrate-electrolyte ingestion enhances cognitive performance during a cross-country mountain biking race
}

\author{
Laura Pomportes ${ }^{1}$, Arnaud Hays ${ }^{1}$, Thibault Gajdos², Jeanick Brisswalter ${ }^{3}$ and Karen Davranche ${ }^{2}$
}

\author{
${ }^{1}$ Aix Marseille Univ, CNRS, ISM, Marseille, France \\ 2 Aix Marseille Univ, CNRS, LPC, Marseille, France \\ ${ }^{3}$ Université Côte d'Azur, Laboratoire Motricité Humaine Expertise Sport Santé, Nice, France \\ * Correspondence: author: Laura Pomportes, Aix-Marseille Université, Faculté de Sciences du Sport, Technosport,163 avenue de Luminy, 13288 , \\ Marseille. laura.pomportes@univ-amu.fr
}

\begin{abstract}
To assess the influence of carbohydrate-electrolyte (CHO-E) intakes on cognitive and physical performances, we carried out an ecological protocol simulating the load variations of a biking race. Eight well-trained athletes completed an outdoor mountain biking time-trial race (TT) $(19.6 \pm 0.40 \mathrm{~km}$ length; $81 \mathrm{~min} \pm 15 \mathrm{~min})$ interspersed with three $10 \mathrm{~min}$ varied-load cycling exercises while performing a Simon task and a Critical Flicker Fusion task in laboratory. A splitted drink of $1150 \mathrm{ml}$ of either 7\% CHO-E solution (fructose $89 \%$, maltodextrin $11 \%$ ) or placebo (PL) was administered. Results suggest that the TT induces a decrease of cortical arousal $(p=0.28)$ and an impairment of efficiency $(p<.001)$ during a decision-making task in the PL condition, while the reverse was observed with CHO-E ingestions. Furthermore, a better accuracy $(p=.05)$ and a lower perceived effort $(p=.05)$ were also observed with $\mathrm{CHO}$ E intakes whatever the TT duration. As a conclusion, ingestion of a 7\% CHO-E solution during a prolonged outdoor TT decreases perceived effort and restricts exercise-induced cognitive performance impairment. CHO-E ingestion appears to be an effective means of delaying the central nervous system fatigue during high-intensity intermittent exercise performed in an ecological context.
\end{abstract}

Keywords: carbohydrates; high-intensity intermittent exercise; nutrition; Simon task; arousal; decision-making task; executive control; prolonged exercise; fatigue.

\section{Introduction}

The use of carbohydrates $(\mathrm{CHO})$ during exercise has received a great deal of attention in recent years, and several reviews suggest an ergogenic effect on performance in a wide range of exercise types and durations $[1,2]$. The guidelines for $\mathrm{CHO}$ intakes by athletes during exercise suggest ingestion from small amounts of $\mathrm{CHO}\left(<30 \mathrm{~g} \cdot \mathrm{h}^{-1}\right)$ or $\mathrm{CHO}$ mouth rinse use for endurance exercise and high-intensity exercise shorter than 45-75 $\mathrm{min}$, and up to $90 \mathrm{~g}$ per hour for ultraendurance events [3]. Several sources of carbohydrates can be consumed such as solid (e.g. bars), semi-liquid (e.g. energy gel) or liquid like $\mathrm{CHO}$-electrolyte beverage, which has become a common choice among both recreational and high-level athletes. In the current scientific literature, the beneficial effect of $\mathrm{CHO}$ feeding on sport performance capacity has been relatively well emphasized. Interestingly, several cognitive components like perception of effort, motor skills and cognitive performance also appear to be sensitive to the ergogenic effects of $\mathrm{CHO}$ ingestion [4]. On this last topic, previous studies have already highlighted a benefits of $\mathrm{CHO}$ intake on information processing during a decision-making task, measured at the end of a submaximal steady-state running exercise shorter than hour [5] or equal to 100-min duration [6].

But to date, the link between $\mathrm{CHO}$ intake and cognitive performance has been less studied during high-intensity intermittent exercise (HIIE) and notably with prolonged duration. This is particularly awkward since many sports have a highly intermittent nature, with bursts of very high intensity bouts followed by relatively low intensity recovery period for a longer duration than 60 minutes (team sports, racket sports, some cycling events...). Interestingly, performance in these sports is heavily dependent on the athlete's capacity to simultaneously handle physiological and cognitive loads under a great temporal pressure. Thus, the prospect of using $\mathrm{CHO}$ intake to enhance cognitive performance is quite appealing since a it has been proposed that supply of blood glucose acts on the brain to enhance functioning [7]. All the more since the exercise-related reduction in circulating blood glucose has been ascribed, while in a nonexclusive manner, within the progressive fatigue occurring during prolonged HIIE [8]. But it important to notice that the transient fatigue that athletes experience during HIIE, mainly due to transitional bursts of very high intensity exercise period involving the contribution of both aerobic and anaerobic metabolic pathways to energy provision could potentially induce negative effect on cognitive performance [9]. Once again, $\mathrm{CHO}$ ingestion could be an interesting 
nutritional strategy to enhance cognitive function since previous studies using the functional magnetic resonance imaging and electroencephalography have shown that the mere presence of $\mathrm{CHO}$ in the mouth activated several brain regions involved in reward and recompense [10,11]. But to date, the few results on this topic in the current literature remain limited and elusive. First, Welsh et al. [12] reported no benefit of $\mathrm{CHO}$ feedings on the Stroop color task performance, which requires inhibition of prepotent responses and selecting relevant sensory information [13], during 60 minutes of shuttle running exercise interspaced with a 20-min halftime passive rest period. On the contrary, a recent study by Dupuy et Tremblay [14] suggests a slight reduction of reaction times throughout exercise with CHO ingestion $(6 \%)$ on cognitive flexibility during a modified Stroop task, measured prior, between and after 3-min bouts of heavy cycling exercise for 36 minutes total duration. It is important to notice that in these two studies cognitive performance was not assessed simultaneously to HIIE exercise, but prior to exercise and during recovery. Furthermore, exercise duration lasted less than 1-hour which can deviate from the reality of the field in several sports.

Based on these observations, we decided to assess the effect of CHO-E solution ingestions on cognitive performance during a prolonged HIIE exercise (i.e. past 1 hour). To that end, we used an ecological context of simulated outdoor cross-country mountain biking race (XCO) which has been previously described like an acyclical and intermittent exercise comparable to high-intensity team sports [15]. Indeed, this discipline requires an intermittent nature of the mechanical workload due to the diverse terrain and pacing characteristics, including both high-intensity and low-speed pedaling phases in the downhill sections. Furthermore, an important cognitive demand is related to motor skill and decisionmaking during races as well as high attentional stress during downhill sections presenting technical difficulties. We decided to create a field-based protocol which promote a best replica of the physiological demand of the race compared to laboratory setting. It includes an explosive rhythm at the start, intermittent bursts, high variability in power, direction speed and cadence in the different portions of each lap, as well as an important physiological load on body muscles due to shock absorption and resisting vibrations during downhill sections [16]. Furthermore, full achievement of exercise in an ecological context induced a greater mental load than those that could be replicable in laboratory. Indeed, various terrains (climbing and downhill sections), pacing strategies and technical difficulties require a continuous attentional focalization throughout the exercise duration (i.e. sustained attention) which could affect cognitive performance in a different way than could have been done in laboratory. Among the diversity of cognitive processes involved in sporting performance, we decided to focus on decision-making and executive control through the Simon task achievement [17]. These processes are essential for our adaptability in an ever-changing environment. We also decided to assess overall central nervous system arousal through a visual sensory-discrimination task called critical flicker fusion task (CFF) [18]. To the best of our knowledge no study has investigated the effect of CHO-E ingestion on arousal and decision processes during HIIE.

Specific hypothesis concerning the way HIIE influences arousal, decision-making performance, and executive control, but also the effect of CHO-E supply before and during HIIE exercise on central fatigue phenomena are posed in this study. We hypothesized that HIIE will induce a cognitive performance impairment, and that the ingestion of $\mathrm{CHO}-$ E drinks would be protective factors of exercise-induced fatigue, especially during the late stages of the exercise (i.e., after about 60-90 minutes of exercise), although a beneficial effect in the earlier bouts of exercise through a centrally mediated effect may also be conceivable.

\section{Materials and methods}

\subsection{Participants}

Twelve well-trained juniors all competing at national and international level took part in this experiment. Due to technical constraints, 4 subjects were excluded from the study. The anthropometric and physiological characteristics of the 8 remaining participants are presented in Table 1. For participants under the age of 18, consent from the legally responsible person has been obtained. Before inclusion, participants were provided with comprehensive written and oral information about the experimental procedures. All participants remained blinded with the use of PL drink in the experimental process. The experimenter simply told them that two different sports drinks were going to be used. The procedure followed was in accordance with the Declaration of Helsinki 1975, revised Hong Kong 1989 and the experimental protocol was approved by the University ethics committee.

Table 1. Anthropometric and physiological characteristics of participants.

\begin{tabular}{cccc}
\hline & \multicolumn{3}{c}{ Mean \pm standard deviation } \\
\hline & All & Male & Female \\
Sample size & 8 & 5 & 3 \\
Age (years) & 17.5 & 17.8 & 17.0 \\
& \pm 1.8 & \pm 2.2 & \pm 1.0 \\
\hline
\end{tabular}




\begin{tabular}{cccc}
\hline Height $(\mathrm{cm})$ & 174 & 179 & 166 \\
& \pm 9.0 & \pm 5.6 & \pm 7.9 \\
\hline Body mass $(\mathrm{kg})$ & 64.0 & 68.7 & 56.0 \\
& \pm 8.2 & \pm 4.2 & \pm 7.3 \\
\hline Fat mass $(\%)$ & 18.2 & 14.4 & 23.3 \\
& \pm 4.5 & \pm 4.9 & \pm 1.6 \\
\hline Heart rate max (bpm) & 198 & 198 & 198 \\
Maximal oxygen con- & \pm 5.2 & \pm 5.2 & 56.6 \\
sumption (ml.min.kg-1) & 61.0 & 67.2 & \pm 2.8 \\
Maximal aerobic power & \pm 8.5 & \pm 4.8 & 265 \\
$(\mathrm{~W})$ & 345 & 393 & \pm 24.0 \\
\hline
\end{tabular}

\subsection{Experimental design}

Participants reported to the laboratory four times over a 4-week period including an incremental exercise test, a familiarization session and two experimental sessions.

\section{Incremental exercise test}

A maximal incremental exercise test was performed on a cyclo-ergometer (Lode Excalibur Sport, Groningen, Netherlands) one month before the experimental sessions. This exercise test started with a 4-minute warm-up at 70 watts (W) for female and $80 \mathrm{~W}$ for male, and then the power output increased by $15 \mathrm{~W}$ per minute until volitional exhaustion. This breaking point was defined as the point where participants stopped or could no longer maintain a pedaling frequency above 50 rotations per minute for more than $10 \mathrm{~s}$ despite a strong verbal encouragement. The last completed step was considered as the maximal aerobic power (MAP). Heart rate (HR) was continuously monitored using a telemetric heart rate (HR) monitor (Garmin, Ltd, Schaffhaussen, Switzerland), enabling assessment of the maximal heart rate $\left(\mathrm{HR}_{\max }\right)$.

\section{Familiarization session}

The day before the first experimental session, participants underwent a familiarization session with the two cognitive tasks: the Simon task and the Critical Flicker Fusion task (CFF).

The Simon task is a reaction time test which involves the processes of inhibition on prepotent responses and selecting relevant sensory information [17]. Participants were faced towards a computer screen located $1 \mathrm{~m}$ away at head height with two thumb response keys fixed on the top of the right and left handle grips. During the task, participants had to fixate on a white point located in the center of the screen and to respond, by pressing the appropriate response key (with the right or left thumb) according to the shape of the stimulus (square or circle) located $7.5 \mathrm{~cm}$ either to the left or to the right of the fixation point. Participants were required to respond as quickly and accurately as possible, according to the shape of the stimulus (task-relevant attribute), while ignoring its spatial location (the task-irrelevant attribute). The stimulus-response mapping (e.g. right/circle and left/square) was counterbalanced across participants. The task included two equiprobable type of trial: the congruent trials (CO) (i.e. the response required corresponds spatially to the location), and the incongruent trials (IN) (i.e. the response required does not correspond to the stimulus location). As soon as a response key was pressed, or when a delay of $1 \mathrm{~s}$ after the stimulus onset had elapsed without a response, the stimulus was removed from the screen and the next trial began. The familiarization with the Simon task consisted of performing a minimum of 3 blocks of 64 trials. Three additional blocks were performed, if needed, until the following learning criteria were achieved: (a) intra-block reaction time (RT) variability below 15\%; (b) inter-blocks RT variability with the previous block below 5\%; (c) mean RT less than $400 \mathrm{~ms}$; (d) error rate between 3\% and 10\%; and (e) positive interference effect (equal to the difference between mean incompatible trials RT and mean compatible trials).

The CFF is a visual sensory-discrimination task that measures the point at which an individual perceives that a flickering light has become fused, and the point at which a fused light begins to flicker [18]. This task is used as an index of overall central nervous activity and cortical arousal and has been shown to be very sensitive to psychotropic drugs [19] and to the exercise-induced changes (e.g. [20]). Participants were seated in a dark and quiet room in front of a viewing chamber device (Flicker Fusion System, model $12021^{*} \mathrm{C}$, Lafayette, United States). Two emitted lights separated by $6.99 \mathrm{~cm}$ and spaced from $38.1 \mathrm{~cm}$ from the eyes, were presented for the right and left eyes simultaneously. Participants were required to respond with their dominant hand by pressing the switch upon when the flicker appeared to fuse (i.e. identification of the visual flicker during ascending trials) and when a fused light appeared to flicker (i.e. fusion threshold during descending threshold). Each trial was alternatively performed with a $1 \mathrm{~Hz}^{-\mathrm{s}^{-1}}$ frequency increment from 0 to $100 \mathrm{~Hz}$ (ascending trial, flicker to fusion) or from 100 to $0 \mathrm{~Hz}$ (descending trial, fusion to flicker). No specific 
learning criterion were applied. The familiarization involved 5 ascending and 5 descending trials to remove practice effects and in order to become familiar with the viewing chamber device. During this familiarization session, participants were also accustomed to the experimental protocol, to the visual analog scale used in the experimental sessions, along with the cycling-accredited racetrack.

\section{Experimental sessions}

The two experimental sessions (CHO-E vs. PL) were held on two consecutive days in a counterbalanced, doubleblind, and cross-over manner. They took place at exactly the same time of the day, to avoid any effect of circadian rhythms, and in similar external environmental conditions (session 1: outdoor temperature $=11^{\circ} \mathrm{C}$ in the morning and $13^{\circ} \mathrm{C}$ in the afternoon, wind speed $=15 \mathrm{~km} \cdot \mathrm{h}^{-1}, 69 \%$ humidity and no precipitation; session 2: outdoor temperature $=13^{\circ} \mathrm{C}$ in the morning and $15^{\circ} \mathrm{C}$ in the afternoon, wind speed $=10 \mathrm{~km} \cdot \mathrm{h}^{-1}, 70 \%$ humidity and no precipitation). Participants were required to refrain from psychostimulant beverages and food (caffeine, guarana, or chocolate) for at least 48 hours before the start of experimental protocol. The same nutrition pattern was maintained before each experimental session. Participants were instructed to refrain from vigorous exercise for 24 hours before the experimental sessions and to sleep at least 8 hours per night the two nights before each session. Except plain water, any ingestion was prohibited in the 90 minutes before the start of experimental sessions.

Each experimental session (overview shown Figure 1) started with a first ingestion, following 30 minutes later by the completion of the CFF task, collection of fingertip blood samples $(\sim 95 \mu \mathrm{L})$ for blood sample analysis and HR monitor fitting. A second ingestion was carried out 40 minutes later, followed by a quick warm-up and a short recall of the Simon task while cycling ( 12 trials). After 5 minutes of rest, the first block of Simon task was performed during a cycling exercise on an ergometer, of varying intensity (see Figure 2 for details). Straight after the end of the exercise, participants went out of the laboratory, activated their GPS position, and started the physical exercise consisting of four laps of an outdoor Cross-Country mountain time-trial race. Ingestions were carried out before the first lap and at the end of each lap. Departure and arrival of each lap were in front of the laboratory entrance to limit the transition time. No rest was allowed during the laps except for the ingestion of drink. After the second and the fourth laps (respectively middle and end of the time-trial race), participants were required to return in the laboratory, provide their ratings of perceived exertion (RPE) and performed the Simon task while cycling on ergometer. Finally, immediately at the end of the experimental session, participants performed the CFF task at rest. The two experimental sessions were strictly identical and lasted approximately $161 \mathrm{~min} \pm 15 \mathrm{~min} 41 \mathrm{~s}$. All measurement realized before the start of the outdoor time-trial race will be described as block 1 (B1), in the middle of the time-trial race as block 2 (B2) and at the end of the time-trial race as block 3 (B3) (Figure 1).

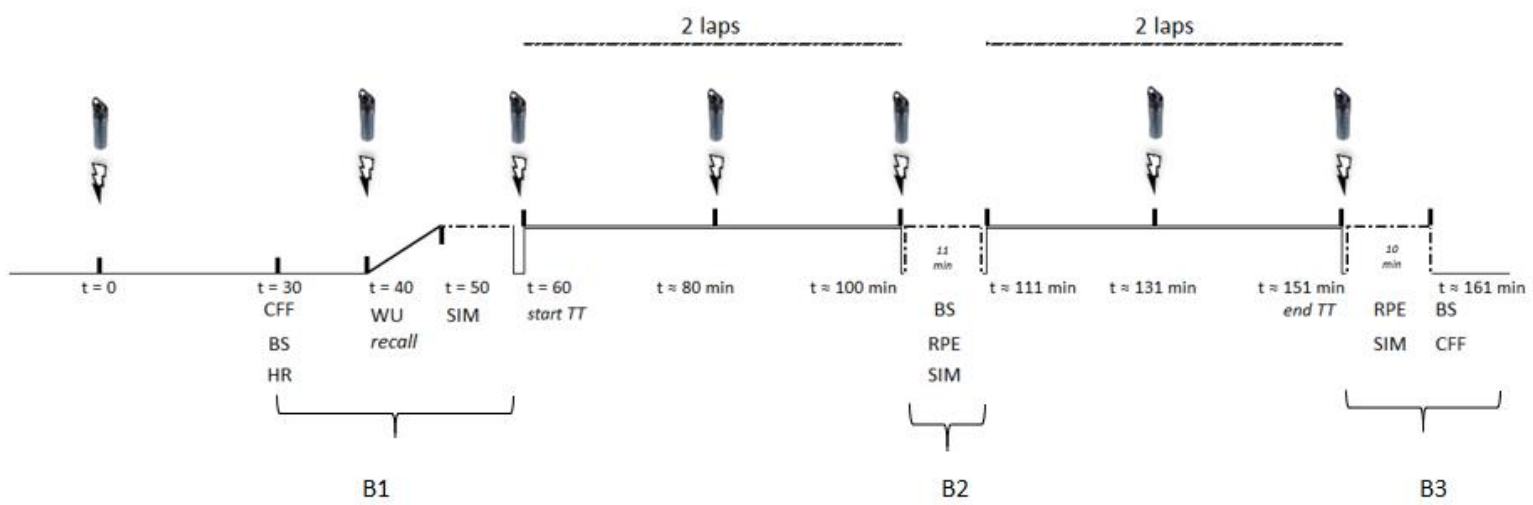

Figure 1. Schematic representation of the general procedure of each experimental session. $\mathrm{CFF}=$ critical flicker fusion task; $\mathrm{BS}=$ blood sample; $\mathrm{HR}=$ heart rate monitoring; $\mathrm{WU}=$ warm up on ergometer; recall = recall of the Simon task; $\mathrm{SIM}=\mathrm{Simon}$ task; TT = time-trial race; $\mathrm{RPE}=$ rating of perceived exertion.

\subsection{Experimental methodology}

Physical exercise and subjective exertion 
The prolonged outdoor time-trial race was performed in the form of a Cross-Country mountain biking race on a cycling track accredited for official competitions. Each run consisted of four laps of $4.9 \pm 0.10 \mathrm{~km}$ length performed on an outdoor track with uphill and downhill sections (cumulative altitude difference for the entire lap $=144 \mathrm{~m}$ ). The total exercise duration of each experimental session was about $112 \min 25 \mathrm{~s} \pm 15 \mathrm{~min} 41 \mathrm{~s}$ excluding the warm-up (9 min). This duration can be further separated into outdoor time-trial race (81 min $25 \mathrm{~s} \pm 15 \mathrm{~min} 41 \mathrm{~s}$ ) and ergometer cycling exercise in the laboratory ( 3 blocks of $10 \mathrm{~min}$ ). Heart rate was continuously monitored throughout the experimental session.

The participant's perceived exertion (RPE) was assessed using a visual analogue scale since it has shown a good reproducibility and sensitivity to assess the perceived exertion during exercise [21]. The scale was completed in the middle of the time-trial (between the second and the third lap, B2) and immediately at the end of the time-trial (after the fourth lap, B3). Participants were required to respond to the following question « What is the perceived exertion of the current effort? », with a temporary marker on a $20 \mathrm{~cm}$ vertical line with two anchor points at the two extremes ("no effort » at the bottom, and « maximal effort » at the top).

\section{Nutritional intervention}

Each participant was given a $1150 \mathrm{ml}$ bolus of either a 7\% carbohydrates-electrolytes solution drink (CHO-E: fructose $(89 \%)$ and maltodextrin $(11 \%)$, salt $(3.4 \mathrm{~g})$, vitamin C (32,5 mg), ISOXAN® Sport Pro, NHS, Rungis, France) or a placebo (PL) artificially colored and flavored but deprived of caloric value. Ingestions were split into seven intakes: i) $250 \mathrm{ml}$ at the start of the experimental session, ii) $150 \mathrm{ml} 40$ minutes later, iii) $150 \mathrm{ml}$ before the start of the time-trial and iv) $4 \times 150 \mathrm{ml}$ apportioned at the end of each of the four laps (Figure 1).

Participants ingested $80 \mathrm{~g}$ of $\mathrm{CHO}$ for about $112 \min 25 \mathrm{~s} \pm 15 \min 41 \mathrm{~s}$ of cycling exercise, which represented a carbohydrate intake of about $43 \pm 6$ g per hour $\left(\mathrm{g} . \mathrm{h}^{-1}\right)$. This rate is consistent with the current nutrition guidelines of 30$60 \mathrm{~g} . \mathrm{h}^{-1}$ for endurance exercise lasting less than 150 minutes [22]. Fluid intake over the two experimental sessions was provided through enforced sports drinks ingestion and plain water which was available ad libitum. The participants were kept blind to the composition of drinks until the end of the study.

\section{Cognitive performance assessment}

During each experimental session, the CFF task was carried out prior to exercise (after the first ingestion, B1) and immediately at the end of exercise (after total ingestion, B3) in a dark and quiet room. Six trials were alternatively performed with a $1 \mathrm{~Hz} \cdot \mathrm{s}^{-1}$ frequency increment from 0 to $100 \mathrm{~Hz}$ (ascending trial, flicker to fusion) or from $100 \mathrm{Ho} 0 \mathrm{~Hz}$ (descending trial, fusion to flicker). Total duration of the task lasted approximately 7 minutes.

In order to be closer to the field reality, the Simon task was performed while cycling on an ergometer with power and duration pedaling changes in order to imitate the performance of a cross-country race (Figure 2). According to the characterization of Cross-Country mountain biking race proposed by Hays et al. [15], we included short-lived and more longer efforts lasting from 30s to 120s and scaled over 50 to $100 \%$ of the MAP. Nevertheless, no suspension period of pedaling commonly described during downhill sections have been included because our experimental protocol does not permit to replicate the physiological load due to shock absorption and the preservation of balance in the downhill phases. The task was performed: before the start of the outdoor time-trial race (B1), in the middle of the outdoor timetrial race (B2) and immediately at the end of the outdoor time-trial (B3) for each experimental session. The establishment of 3 blocks allows to disentangle the effect of HIIE ( $<1$ hour) and prolonged HIIE on cognitive performance (respectively B1 vs. B2 and B1 vs. B3). Each block included 304 trials interspaced with a $500 \mathrm{~ms}$ inter-stimulus interval. For the purpose of steady pedaling parameters, the Simon task started after $45 \mathrm{~s}$ of cycling exercise on ergometer and lasted approximately 9 minutes $15 \mathrm{~s}$.

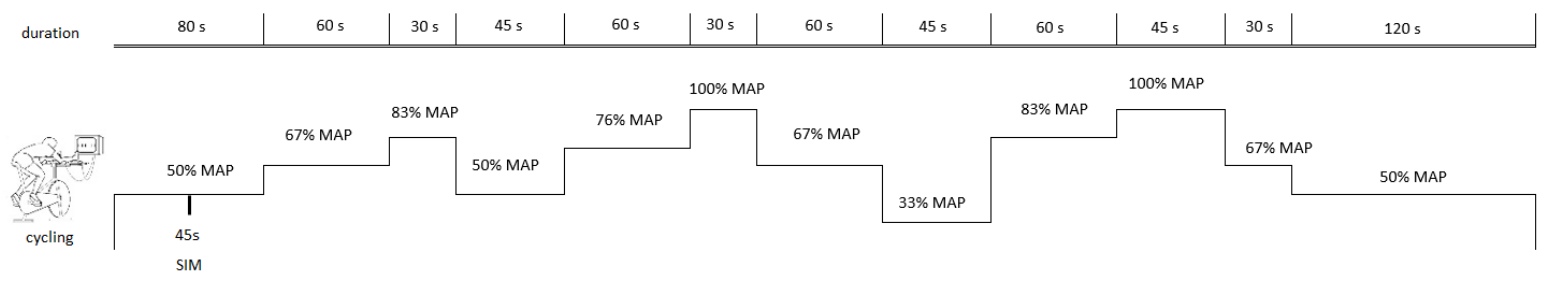

Figure 2. Schematic representation of the intensity and duration of ergometer cycling exercise during Simon task. MAP $=$ maximal aerobic power, SIM = Simon task.

Blood sample analysis 
Capillary blood samples were immediately analyzed for glycemia, $\mathrm{pH}$, bicarbonate, potassium, and sodium (iSTAT clinical analyzer, Abbott Point of Care, East Windsor, NJ, United States). HR, GPS position and time performance were continuously recorded throughout the outdoor time-trial race (Garmin Edge 520 Ltd., Schaffhausen, Switzerland).

\subsection{Data treatment and Statistical analysis}

After exclusion of 4 subjects, the 8 remaining participants' data was analyzed according to the following procedure.

\section{Cognitive performance}

For the CFF task, the mean value of the six trials, representing the sensory sensitivity criterion ( $\left.\mathrm{M}_{\mathrm{tot}}\right)$, was calculated for each participant. In addition, the subjective judgment criterion (Mdi) was calculated, by subtracting the mean value of the ascending trials to the mean value of the descending trials. We analyzed $\mathrm{M}_{\text {tot }}$ and $\mathrm{M}_{\mathrm{di}}$ within a hierarchical linear mixed-effects model, including drink (CHO-E, PL), exercise (B1, B3) and their interactions as fixed effects, and the intercept, drink, exercise random effects.

Results in the Simon task were analyzed across three dimensions. First, performance was evaluated by response accuracy (error rate), speed (reaction time, RT) as well as efficiency rate and coefficient of variation of reaction time. We analysed (logarithm of) RTs within a hierarchical linear mixed-effects model, including drink, exercise duration (B1, B2, $\mathrm{B} 3)$, congruence $(\mathrm{CO}, \mathrm{IN})$, accuracy (correct, error) and their interactions as fixed effects, and the intercept, drink, exercise duration, congruence and response accuracy as random effects. Response accuracy was also analyzed within a hierarchical generalized linear mixed-effects model, including the drink, exercise duration, congruence, (centred) RT (in seconds) and their interactions as fixed effects, and the intercept, drink, exercise duration, congruence, and RT as random effects. Efficiency rate was defined by accuracy ("accuracy" takes a value of 1 if the trial is correct, and 0 otherwise) divided by RT (expressed in seconds). We analyzed efficiency within a hierarchical linear mixed-effects model, including, exercise duration, drink and their interactions as fixed effects, and the intercept, drink, exercise duration as random effects. We also analyzed coefficient of variation of reaction time within a hierarchical generalized linear mixedeffects model, including the drink, exercise duration and the interactions as fixed effects, and the intercept, drink, exercise duration as random effects.

Secondly, the impulsivity level was evaluated by the Error Location Index (ELI), which measures the proportion of fast errors that are located among the fastest trials. We computed the Error Location Index by subject and by block (exercise duration) for each condition. We then estimated a hierarchical mixed-effect model, block (coded with repeated contrasts), drink and their interaction as fixed effects, and a random intercept.

Finally, inhibition ability was evaluated by the Interference Dynamics Index (IDI), which measures the concentration of the interference effect (i.e., the difference between RTs of IN and CO trials) among the fastest trials [23]. We computed the Interference Dynamics Index by subject and by block for each condition. We then estimated a hierarchical mixed-effect model using block (coded with repeated contrasts), drink and their interaction as fixed effects, and a random intercept.

Predictors were coded using sum contrasts (thus "accuracy" takes a value of 1 if the trial is correct, and - 1 otherwise, "congruent" takes a value of -1 for congruent trials and 1 otherwise; "drink" takes value of 1 when participants received $\mathrm{CHO}-\mathrm{E}$, and -1 otherwise). The "duration" variable is the (mean-centered) rank of each trial and is interpreted as a proxy for exercise duration. Finally, RTs, when used as an explanatory variable, were mean-centered and expressed in seconds. Both data were analyzed using the lmer4 package [24] in R (version 4.2, R Core Team, 2017). All regressions were performed with the restricted maximum likelihood fitting method, and $p$ values for coefficients were obtained with the lmerTest package [25].

\section{Subjective exertion and physical performance}

RPE score was determined by the distance in centimeters from the bottom extreme anchor line to the horizontal participant's mark. We analyzed ratings of perceived exertion, within a hierarchical generalized linear mixed-effects model, including the drink and exercise duration (B2, B3) (centred) and their interactions as fixed effects, and the intercept, drink, exercise duration as random effects.

Concerning physical performance, we analyzed time performance within a hierarchical generalized linear mixedeffects model, including the drink and laps (lap 2, lap 3 and lap 4) expressed in percentage of the first lap and their interactions as fixed effects, and the intercept, drink, laps as random effects.

\section{Blood analysis and heart rate}

Blood glucose was expressed in absolute value and was then estimated a hierarchical mixed-effect model, exercise duration (B1, B2, B3) (coded with repeated contrasts), drink and their interaction as fixed effects, and a random intercept. 
Mean of heart rate was expressed in percentage of the maximal heart rate. We then estimated a hierarchical generalized linear mixed-effects model, including the drink and laps (lap 1, lap 2, lap 3 and lap 4) and drink and their interaction as fixed effects, and a random intercept.

\section{Results}

\subsection{Cognitive performance}

Critical Flicker Fusion task

Concerning the sensory sensitivity criterion (Mtot) results showed an interaction between drink and exercise $(\beta=$ $.37, \mathrm{SE}=.13, \mathrm{p}=.028$ ), with decrease of $\mathrm{M}_{\text {tot }}$ after exercise in the PL condition (PLB1 $=40.63 \pm 3.91 \mathrm{~Hz}$ vs. PLb3 $=39.38 \pm$ $3.21 \mathrm{~Hz}, \Delta=1.25 \mathrm{~Hz}$ ), while no change has been observed in the CHO-E condition (CHO-Eв1 $=40.03 \pm 2.71 \mathrm{~Hz}$ vs. CHO$\mathrm{E}_{\mathrm{B} 3}=40.23 \pm 2.89 \mathrm{~Hz}, \Delta=0.20 \mathrm{~Hz}$ ) (Figure 3). This impairment of the sensory sensitivity criterion under placebo affects six out of eight participants.

Results of the subjective criterion ( $\mathrm{M}_{\mathrm{di}}$ ) suggested no significant main effect or interaction in the subjective judgement of participants (drink: $\beta=.36, \mathrm{SE}=.55, \mathrm{p}=.53$; exercise: $\beta=.61, \mathrm{SE}=.51, \mathrm{p}=.25$; drink X exercise: $\beta=-0.44, \mathrm{SE}=$ $.51, \mathrm{p}=.40)$.

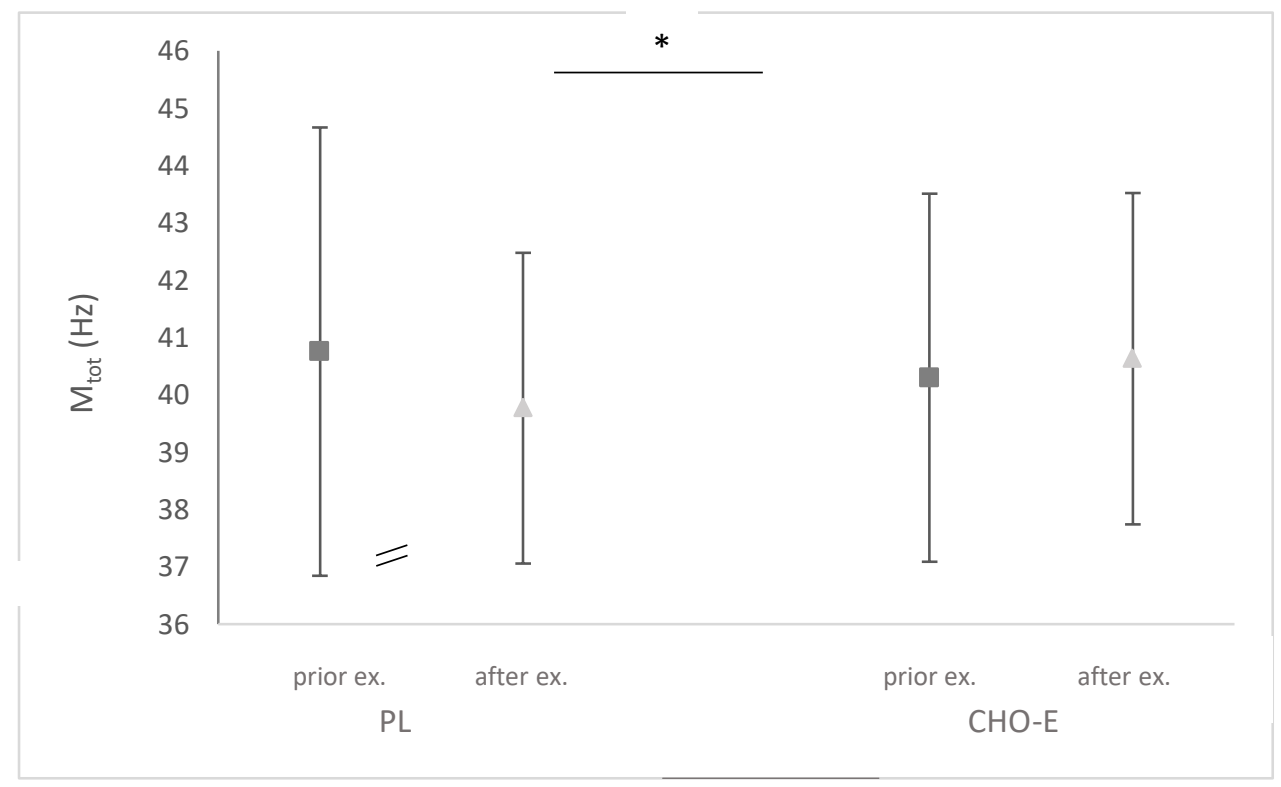

Figure 3. Sensory sensitivity threshold criterion (Mtot) with PL and CHO-E conditions prior exercise (B1) (full squares) and after exercise (B3) (full triangles). Error bars represent standard deviation of the mean. ${ }^{*} \mathrm{p}<.05$.

\section{Simon task Efficiency}

We observed an interaction between drink and exercise duration $(\beta=.11, \mathrm{SE}=.0019, \mathrm{p}<.001)$ suggesting an increase of efficiency after exercise (B3) compared to middle exercise (B2) under CHO-E condition, whereas a decrease of efficiency is found under PL ingestion (PLв2: $2.55 \pm 1.2$ vs. PLвз: $2.44 \pm 1.1 ; \Delta=-4.3 \%$; СНО-Ев2: $2.52 \pm 1.1$ vs. СНО-Евз: 2.63 $\pm 1.1, \Delta=+4.4 \%)$.

\section{Reaction time}

We observed a classical effect of congruence $(\beta=.023, \mathrm{SE}=.0022, \mathrm{p}<.001)$, with slower response time for incongruent than congruent trials (CO: $405 \pm 90 \mathrm{~ms}$ vs. IN: $422 \pm 88 \mathrm{~ms}, \Delta=17 \mathrm{~ms}$ ). We have also found a negative interaction between exercise duration and drink $(\beta=-.0057, \mathrm{SE}=.0014, \mathrm{p}<0.001)$, showing that RTs increase at the end of exercise compared to middle exercise in the PL condition, while the converse holds under CHO-E ingestion (Figure 4) (PLв2: 411 \pm 97 ms vs. РLвз: $423 \pm 87 \mathrm{~ms}, \Delta=+12 \mathrm{~ms}$; СНО-Ев2: $418 \pm 100 \mathrm{~ms}$ vs. СНО-Евз: $400 \pm 98.3, \Delta=-18 \mathrm{~ms})$. The same interaction tended to be significant in the middle exercise (B2) compared to before exercise (B1) $(\beta=-.010$, SE $=.0053, \mathrm{p}=.06$; PLB1: $404 \pm 75 \mathrm{~ms}$ vs. PLв2, $\Delta=+7 \mathrm{~ms}$; СНО-Ев1: $425 \pm 99 \mathrm{~ms}$ vs. СНО-Ев2, $\Delta=-7 \mathrm{~ms}$. None of the other independent variables were significantly correlated with RT (all ps $>.08$ ). 


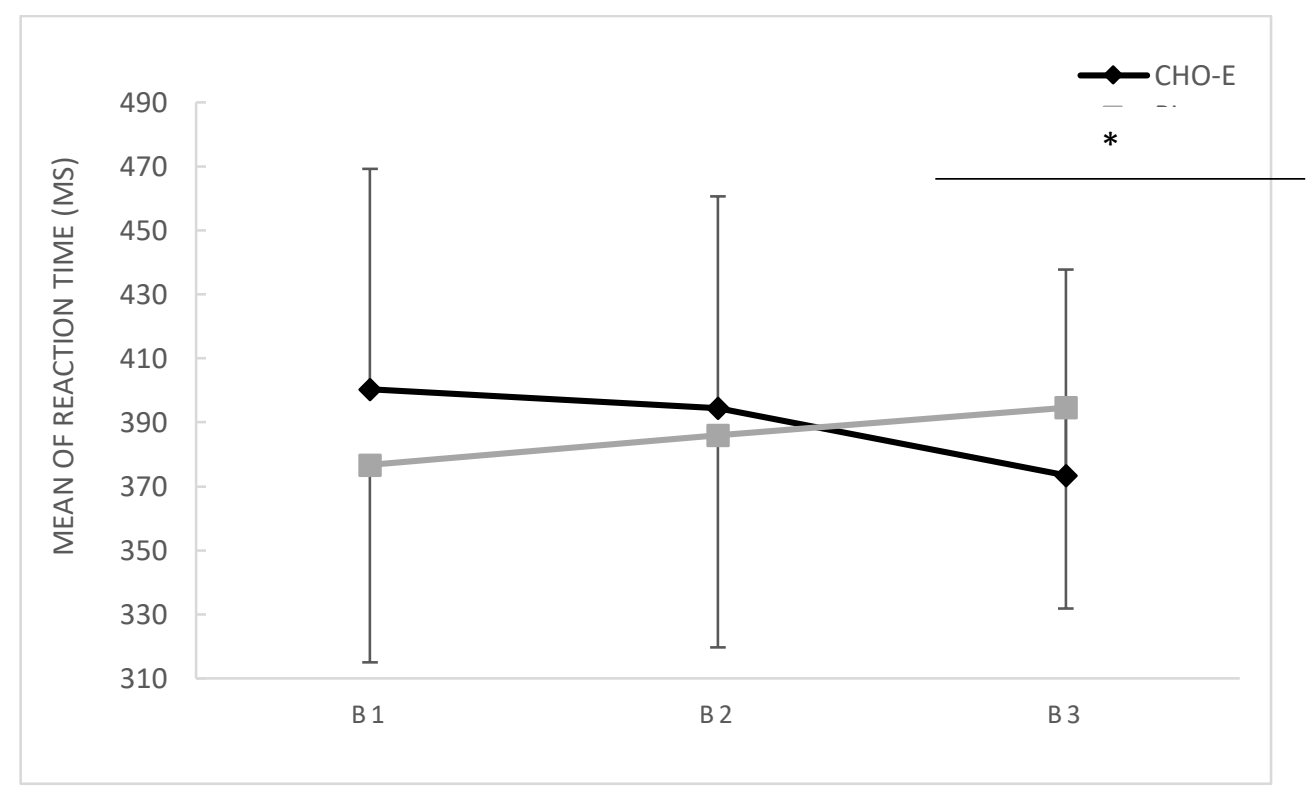

Figure 4. Reaction time performance (ms) for each block (B1, B2 and B3) and each condition (CHO-E, PL). Error bars represent standard deviation of the mean divided by two. ${ }^{*} \mathrm{p}<.05$.

Coefficient of variation of reaction time

We observed a significant effect of drink with lower variability under CHO-E condition compared to PL whatever the time duration of exercise $(\beta=-.011, \mathrm{SE}=.0032, \mathrm{p}=.0020 ; \mathrm{CHO}-\mathrm{E}: 31 \pm 10 \%$ vs. PL: $33 \pm 7 \%)$.

\section{Response accuracy}

We observed a classical effect of congruence, with higher error rate for incongruent trials compared to congruent trials $(\beta=-.17, \mathrm{SE}=.0685, \mathrm{p}=.042 ; \mathrm{CO}: 5.3 \pm 3 \%$ vs. IN: $7.1 \pm 4 \%)$. Furthermore, error rate increased in the middle of exercise (B2) compared prior to exercise (B1), regardless of drink $(\beta=-.021, \mathrm{SE}=.089, \mathrm{p}=.016$; B1: $5.4 \pm 3.2 \%$ vs. B2: 6.7 $\pm 4.4 \%$ ) (Figure 5a). Finally compared to PL error rate, the $\mathrm{CHO}-\mathrm{E}$ condition tended to be lower whatever the time exercise duration $(\beta=.143 \mathrm{SE}=.068, \mathrm{p}=.050$; CHO-E: $6.3 \pm 4.3 \%$ vs. PL: $8 \% \pm 5.3 \%$ ) (Figure $5 \mathrm{~b}$ ).
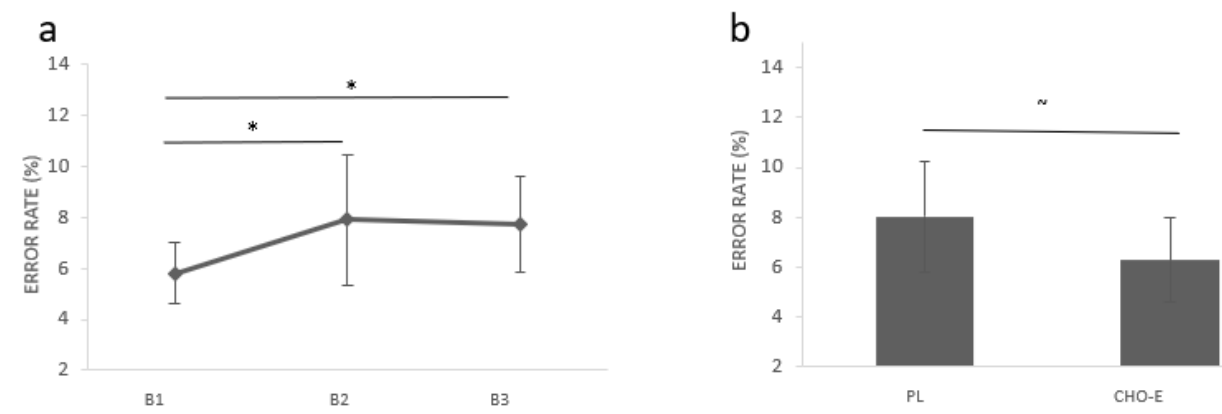

Figure 5: Effect of exercise duration (Figure 5a) and condition (Figure 5b) on the response accuracy. Errors bars represent standard deviation of the mean divided by two. ${ }^{*} \mathrm{p}<.05 ; \sim \mathrm{p}=.05$.

Impulsivity

None of the variables were significantly correlated to ELI (all ps>.51).

Inhibition

None of the variables were significantly correlated to IDI (all ps>.13).

3.2. Subjective exertion, physical performance, and blood sample 
Time durations for the first and second half of the TT race were respectively $40 \mathrm{~min} 30 \mathrm{~s} \pm 7 \mathrm{~min} 30 \mathrm{~s}$ (laps 1 and 2) and $43 \mathrm{~min} 40 \mathrm{~s} \pm 10 \mathrm{~min} 6 \mathrm{~s}$ (laps 3 and 4) and apportioned as follows : lap 1: $20 \mathrm{~min} 05 \mathrm{~s} \pm 4 \mathrm{~min}$; lap 2: $20 \mathrm{~min} 25 \mathrm{~s} \pm 4$ min; lap 3: $21 \mathrm{~min} 20 \mathrm{~s} \pm 5 \mathrm{~min}$; lap 4: $22 \mathrm{~min} 20 \mathrm{~s} \pm 5 \mathrm{~min}$. Time duration of the outdoor time-trial race was about $84 \mathrm{~min}$ $10 \mathrm{~s} \pm 17 \mathrm{~min} 10 \mathrm{~s}$ with an important gender difference (female: $103 \mathrm{~min} 53 \mathrm{~s} \pm 6 \mathrm{~min} 15 \mathrm{~s}$; male: $70 \mathrm{~min} 55 \mathrm{~s} \pm 7 \mathrm{~min} 23$ s).

Ratings of perceived exertion

A main effect of drink has been observed $(\beta=-0.58, \mathrm{SE}=0.26, \mathrm{p}=.05)$ with lower RPE in the CHO-E condition compared to the PL condition whatever the time-trial race duration ( $\mathrm{CHO}-\mathrm{E}=16.9 \pm 2.23 \mathrm{vs}$. $\mathrm{PL}=17.8 \pm 2.4, \Delta=0.9)$. No interaction between drink and exercise duration has been shown $(\beta=-0.43, \mathrm{SE}=0.27, \mathrm{p}=.13)$.

Time performance

A significant impairment of the time performance has been found in lap $3(+6.67 \pm 10 \%)$ compared to lap $2(+1.3 \pm$ $4 \%)(\beta=5.37, \mathrm{SE}=1.89, \mathrm{p}=.03)$, and tended to be significant in lap $4(+11 \pm 17 \%)$ compared to lap $3(\beta=4.26, \mathrm{SE}=2.03, \mathrm{p}$ $=.07$ ). No interaction effect between drink and laps has been observed: (drink X laps 2 and 3: $\beta=-1.6, \mathrm{SE}=1.79, \mathrm{p}=.40$; drink X laps 3 and 4: $\beta=-3.3, \mathrm{SE}=2.4, \mathrm{p}=.21$ ) (Figure 6).

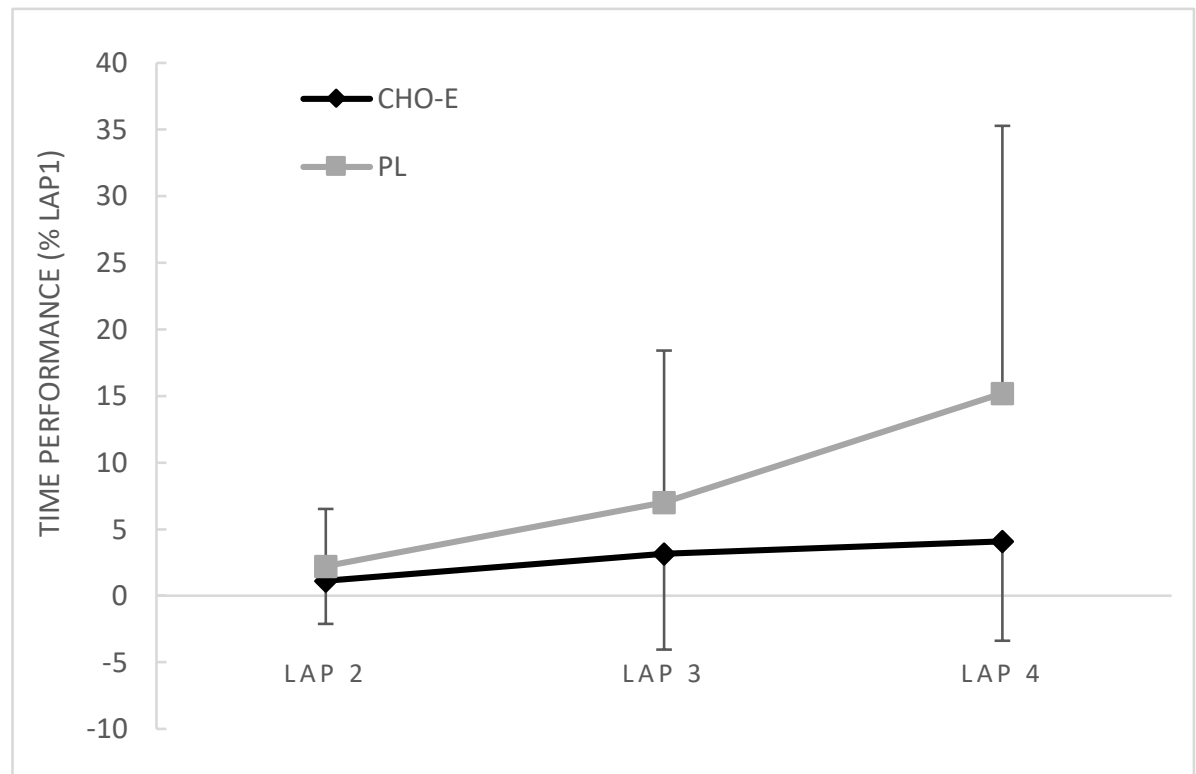

Figure 6. Time performance for each lap of the time-trial race (expressed in \% of the lap 1) with PL (full squares) and CHO (full circles) conditions. Error bars represent standard deviation of the mean.

\section{Heart rate}

Mean heart rate was $83.5 \pm 6.4 \% \mathrm{HR} \max$ during the time-trial with no difference between PL and CHO-E condition. However, mean heart rate in lap 3 was significantly lower compared to lap $2(\beta=-4.02, \mathrm{SE}=1.3, \mathrm{p}=.01)$ and tended to be higher compared to lap 4 ( $\beta=-0.98, \mathrm{SE}=0.49, \mathrm{p}=.08$; lap 1: $86.4 \pm 4.9 \% \mathrm{HR}_{\max }$ lap 2: $85.3 \pm 5.9 \%$ HRmax, lap 3: $81.7 \pm$ $5.7 \% \mathrm{HR}_{\max }$, lap 4: $\left.80.6 \pm 7.4 \% \mathrm{HR} \max \right)$. No interaction between drink and laps have been shown (p>.29).

Blood sample

As expected blood glucose was significantly higher in the CHO-E condition compared to PL condition ( $\beta=0.21$, $\mathrm{SE}=0.08, \mathrm{p}=.04$ ) whatever the exercise duration (CHO-E: $5.46 \pm 0.8 \mathrm{mmol} . \mathrm{l}^{-1} \mathrm{vs}$. PL: $5.04 \pm 0.6 \mathrm{mmol} . \mathrm{l}^{-1}, \Delta=0.42 \mathrm{mmol} .1$ 1). Furthermore, a significant decrease of blood glucose has been found at the end of exercise (B3) compared to middle exercise (B2) $\left(\beta=-70.7, \mathrm{SE}=0.2, \mathrm{p}=.006\right.$; B2: $5.43 \pm 0.7 \mathrm{mmol.1^{-1 }}$ vs. B3: $\left.4.7 \pm 0.6 \mathrm{mmol} . \mathrm{l}^{-1}, \Delta=0.73 \mathrm{mmol.} \mathrm{l}^{-1}\right)$ but without any significant difference between CHO-E and PL condition $\left(\beta=-2.7^{-17}, \mathrm{SE}=0.1, \mathrm{p}=1\right.$; CHO-Ев2: $5.7 \pm 0.8 \mathrm{mmol.1} \mathrm{I}^{-1}$; CHOЕвз: $5 \pm 0.6$ mmol.1-1; PL в2: $5.1 \pm 0.3$ mmol..$^{-1}$; PLвз: $\left.4.3 \pm 0.5 \mathrm{mmol} . \mathrm{l}^{-1}\right)$. No significant difference between B1 and B2 has been found in blood glucose level $\left(\beta=0.18, \mathrm{SE}=0.3, \mathrm{p}=0.58\right.$; B1: $\left.5.6 \pm 0.6 \mathrm{mmol}^{-1}\right)$ (Figure 7). 


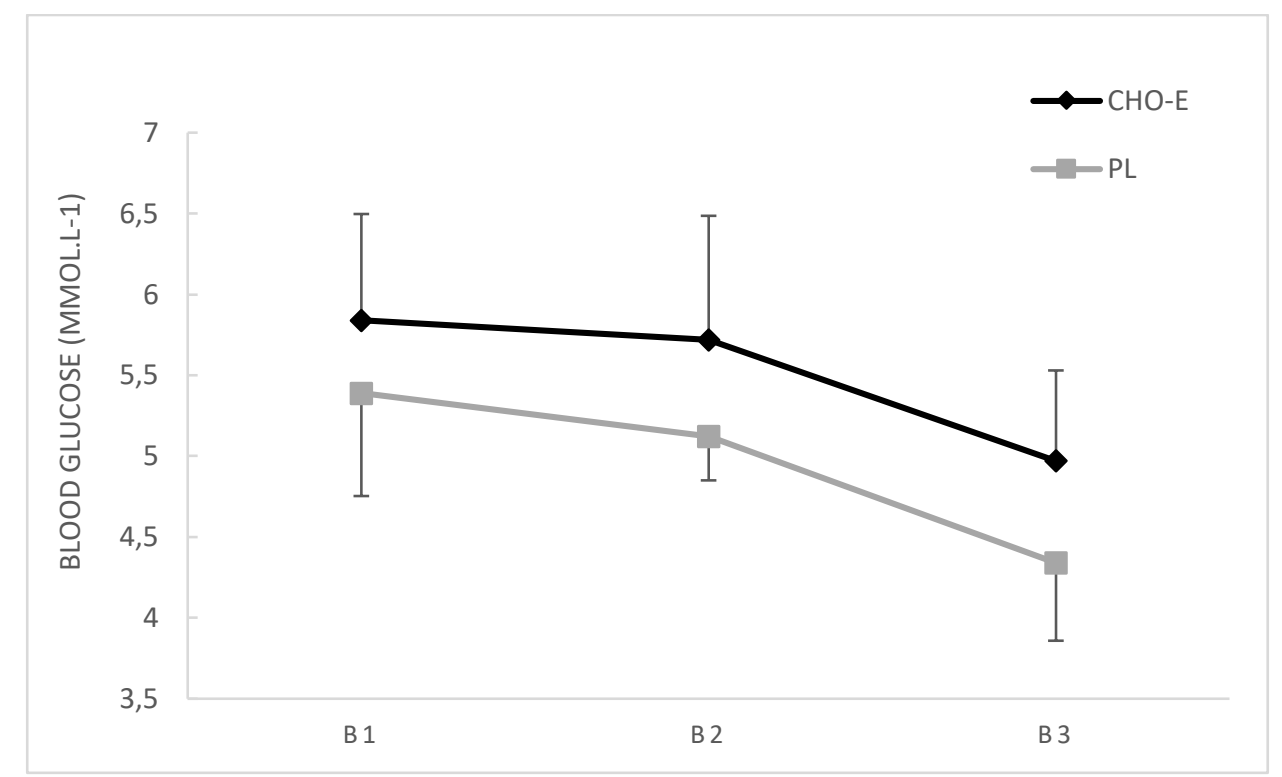

Figure 7. Change in blood glucose level during experimental sessions in PL (full squares) and CHO (full circles) conditions. Measures in block 1 were performed before exercise, block 2 in the middle of exercise and block 3 after exercise. Error bars represent standard deviation of the mean.

\section{Discussion}

In the current study, participants performed a prolonged high-intensity intermittent exercise (HIIE) in an ecological context with PL or CHO-E ingestions. HIIE was performed in the form of a cross-country mountain biking race (XCO) on an outdoor cycling track accredited for official competitions. An original aspect of our study is that cognitive performance was assessed simultaneously to cycling exercise in laboratory but with varying intensity which is closer to the reality on the ground compared to steady-state exercise. The main findings of this study are that ingestions of a $1150 \mathrm{ml}$ bolus of 7\% CHO-E drink before and during outdoor time-trial races enhance accuracy and lower perception of effort in the earlier phases of HIIE. Additionally, ingestions of CHO-E solution have the ability to restrict impairment of information processing efficiency and cortical arousal decrement, both occurring in the late phases of HIIE.

Regarding to the critical flicker fusion task (CFF), the results reported here suggest an exercise-induced impairment of sensory sensitivity criterion $\left(\mathrm{M}_{\mathrm{tot}}\right)$ without any effect on subjective judgment criterion $\left(\mathrm{M}_{\mathrm{di}}\right)$. These results can be interpreted as a decrement of cortical arousal induced by prolonged HIIE, whereas decision factors remain unaffected. To the best of our knowledge, no study has investigated the effect of prolonged HIIE on cortical arousal using CFF task. The few studies on this topic, have been performed during prolonged submaximal steady-state exercise. To date, results remain inconsistent between those which suggest an increased level of cortical arousal after cycling exercise to exhaustion at $70 \% \dot{V} O_{2 \max }$ [26], and those which report an alteration of subjective judgment criterion in the form of more risky approach from 120 minutes cycling exercise at $60 \% \dot{V} O_{2 \max }$ [27]. Interestingly, our results suggest that CHO-E ingestion has a profound effect on the central nervous system function since a maintaining of cortical arousal ( $\mathrm{M}_{\text {tot }}$ ) has been reported under CHO-E condition. This result supports a previous study having reported a beneficial effect of CHO-E ingestion on self-reported arousal, using a Felt Arousal Scale during a prolonged HIIE designed to simulate the minimum physiological demands experienced by soccer players [28]. The maintenance of sensory sensitivity with ingestion of a CHO-E solution highlights a limitation of central nervous system arousal decrement and can explained, at least in faction, by the facilitation of information processing during the Simon task. Indeed, previous studies have already proposed a link between cortical arousal, the responsiveness of sensory systems to various environmental stimuli and consequently to information processing and cognitive functions [18,29]

A decrement of information processing efficiency and RT performances in the PL condition after the full completion of the TT race have been highlighted in this study (respectively $\Delta=-4.3 \%$ and $\Delta=+12 \mathrm{~ms}$ ). These findings, which can be interpreted as a behavioral manifestation of central nervous system fatigue, are in accordance with a recent study stemming from the military field. Eddy et al. [30] reported a decrement of speed of information processing during a modified Go/Nogo task (another response inhibition task) in the second hour of the prolonged march with a load carry (40 kg) compared to unloaded condition. Once again, in our study, nutritional intervention seems worthwhile since efficiency increases with CHO-E intakes after full outdoor time-trial race completion (B3, about 100 minutes of exercise) compared to the middle of the race (B2). This result agrees with a previous study which lends some support to a positive effect of $\mathrm{CHO}$ ingestion on maintenance of visual reaction time performance following 80 minutes of HIIE exercise in 
squash players [31]. More precisely, results of this last study highlighted an increase of visual RT occurring after HIIE in the PL condition, whereas the opposite has been found under $\mathrm{CHO}$ condition. Interestingly our results highlighted a lower variability for reaction time and a better accuracy (without a speed-accuracy trade-off) in the CHO-E condition compared to PL condition whatever the TT race duration. These findings support a real cognitive performance improvement through enhancement of attentional and/or perceptional processes in the earlier bout of HIIE under CHO-E condition. This beneficial effect, which occurs in the earlier phases of HIIE performed when carbohydrate availability for energy production do not decrease and in a fed state, could imply a centrally mediated mechanism. Previous study has already raised the possibility of taste receptors located in the mouth which could influence neural pathways [32] and subsequent cognitive performance.

One issue needs to be noted, however. Although our data highlights that information processing efficiency remain fully efficient in the first half of the time-trial race (B2, after $40 \mathrm{~min} 30 \mathrm{~s} \pm 7$ min $30 \mathrm{~s}$ of time-trial race), this result must be treated carefully since the analysis of accuracy data suggest an increase of error rate during the same period, regardless of the drinks ingested. Given these results, it appears that fatiguing characteristics of the HIIE, mainly due to a high physiological load $(83.5 \pm 6.4 \% \mathrm{HR} \max$ in our study), transitional bursts of very high intensity exercise period, and the contribution of both aerobic and anaerobic metabolic pathways to energy provision could induce a negative effect on cognitive performance in the earlier bout of exercise. This notion has been supported by findings from Dupuy and Tremblay [14] who claimed a decrease of RT and accuracy during a cognitive flexibility task towards the end of $6 \times 3$ minutes of heavy cycling exercise. Some neuroendocrinological model issued explanations for this cognitive performance impairment. It has been proposed that during high level of stress, a higher activation of the sympathoadrenal system as that already stimulates during moderate exercise, and the release of HPA hormones axis like cortisol could add to the negative effects of catecholamines and contribute to the cognitive performance impairment [9]. But it must be emphasized that our results are not supported unanimously since other studies reported a beneficial effect of a short volume of HIIE ( $\leq 33$ minutes) on speed of information processing [33,34]. Two main factors could explain these discrepancies. First, in our study, the Simon task was performed simultaneously to cycling exercise with variable-load which is closer to the reality field with up and down hills but increases the attentional demands and so dual-task cost upon physical exercise. Additionally, it is important to note that the ecological context of prolonged HIIE increased the mental load due to maintenance of balance in the technical parts of the race and a high risk of falling compared to laboratory settings which could induce additional fatigue.

Interestingly, our results suggest that neither intensity (HIIE $<1$ hour) nor prolonged duration (HIIE $>1$ hour) deteriorated selective inhibition processes and impulsivity. This first finding is consistent with previous study having suggested a robustness of these processes during a Simon task after continuous short duration but high-intensity exercise [35]. Furthermore, no additional effect of CHO-E ingestions has been highlighted in this study. This outcome is in accordance with Welsh and collaborators [12] who reported no effect of CHO-E ingestion on inhibition processes during a Stroop Color-Word Test throughout 60 minutes of HIIE. However, more recently, Dupuy and Tremblay [14] suggested a beneficial effect of $\mathrm{CHO}$ beverage $(6 \%, 60 \mathrm{~g})$ on cognitive flexibility tasks, another executive control function, measured after short duration HIIE. Several methodology discrepancies and moderator variables including when cognition is measured, and the type of cognitive task [36] could limit generalization of $\mathrm{CHO}$ ingestion on executive control during exercise.

Although this study was not designed to determine underlying mechanism(s) of CHO-E benefits, several hypotheses have been made. We first suggested that $\mathrm{CHO}$ feeding could be related to increased or at least maintained substrate to the brain during HIIE. Indeed, failure of glucoregulation induced by prolonged exercise may trigger a subsequent hypoglycemia involving several cognitive performance impairments including decrement of sensory processing abilities and decision-making performance [37]. However, in this study, we observed a decrease in blood glucose concentration in the late stages of exercise, but no significant difference between the PL and CHO-E conditions has been found (PLвз: $4.3 \pm 0.5 \mathrm{mmol}^{-1}{ }^{-1}$ СНО-Евз: $5 \pm 0.6 \mathrm{mmol}^{-1}{ }^{-1}$. Additionally, the control trial did not result in hypoglycemia since blood glucose concentration after the TT race (112 $\min 25 \mathrm{~s} \pm 15 \mathrm{~min} 41 \mathrm{~s})$ was $4.3 \pm 0.5 \mathrm{mmol}^{-\mathrm{l}^{-1}}$, which is higher than hypoglycemia threshold i.e., 2.5 mmol.1-1 [38]. It appears that euglycemia has been maintained until the late course of HIIE and consequently, that the mechanism(s) underlying cognition-enhancing effects of $\mathrm{CHO}$ are unlikely to be mediated by a metabolic effect. A strong assumption to consider is the involvement of a centrally mediated mechanism to explain the benefits of CHO-E ingestion. Previous neuroimaging investigations have already shown that the mere presence of $\mathrm{CHO}$ in the buccal cavity produced an increase of cortical activation when compared with a PL solution [11,32]. Our results offer clear arguments in favor of this assumption, since a maintenance of sensory sensitivity criterion ( $\left.\mathrm{M}_{\mathrm{tot}}\right)$, regarded as an indirect measure of cortical arousal, has been found in the $\mathrm{CHO}$ condition after the TT race. More specifically, activation of T1RS receptors located in the mouth, induced by the caloric value of the solution have shown to 
increase brain activity in reward and motivation centers. The behavioural manifestations of this increase of activation could be beneficial for brain functioning, enhancing both physical and cognitive performance.

Not without interest, a lowered perceived exertion during the whole HIIE has been highlighted in our study under $\mathrm{CHO}-\mathrm{E}$ condition. This finding which is in accordance with previous studies on this topic (e.g. [39]) is worthwhile since in a real competition condition, any decrease in perceived exertion could improve performance. Among the possible explanations of this ergogenic effect, we can suggest a centrally mediated effect. Without being the exclusive hypothesis, it is also conceivable that the metabolic effect of carbohydrate and notably the maintenance of glycogen stores in the muscles, influences perception of effort through feedback from group III and IV muscle afferents, which constitutes the sensory signal involved in perception of effort generation [40]. Thus, CHO-E feeding could induce a differing tolerance of pain/discomfort, perhaps positively influence sensations of lethargy, tiredness, and motivation, and so decrease perception of effort.

Concerning physical performance, the HIIE performed in the form of a cross-country mountain biking race induces physiological state of fatigue. This statement is attested by a decrease of performance over consecutive laps, and a decrease of blood glucose in the last bouts of exercise. Several explanations have been implied in the generation of fatigue that riders experienced under such pattern of HIIE. First, a transient fatigue could be associated with a high anaerobic energy turnover resulting in increased concentration of inorganic phosphate, a decrease of $\mathrm{pH}$, disturbances in the muscle sodium and potassium homeostasis or failure in the calcium release process [8]. Our results seem to confirm this direction since a decrease of $\mathrm{pH}$ and an increase of extracellular potassium has been highlighted in our study (data not presented). Additionally, depletion of muscle glycogen stores and down regulation of blood glucose have been proposed in the aetiology of fatigue in the last stage of HIIE [41]. Rationally, CHO ingestions appear to be an interesting nutritional strategy to postpone fatigue, and experimental contributions have emphasized the role of $\mathrm{CHO}$ ingestion to reduce muscle glycogen use, spare reserves [42] and avoid impairment of neuromuscular transmission or muscle processes. Surprisingly, our results do not suggest a beneficial effect of CHO-E intakes on physical performance. This finding is not in accordance with plentiful experimental data suggesting an ergogenic effect of $\mathrm{CHO}$ feeding on long duration and constant-load exercise (e.g. [43]). But then, it is true that during HIIE, where there are multiple performance factors, notably, the major impact of motor skills, the ergogenic effect of $\mathrm{CHO}$ on physical performance would be far more difficult to establish and especially during an ecological protocol. Additionally, the weak sample size could be involved in the lack of a beneficial effect of $\mathrm{CHO}$ ingestion on physical performance during prolonged HIIE.

\section{Limitations}

The present study has several limitations. Firstly, the small sample size, strengthened by the exclusion of 4 participants ( 2 during experimental sessions and 2 during data processing), is an important limitation in this study. This may be due to the ecological character of our study, but also to the difficulty in recruiting well-trained subjects competing at national and international level. Moreover, in the current study the CHO-E and PL drinks condition were not identical in electrolyte concentration (sodium principally) and vitamin C. Thus, we were unable to comprehensively separate the relative contribution of carbohydrates and electrolytes or vitamin $C$ in the beneficial effects.

\section{Conclusion}

In conclusion, our findings contribute to growing evidence that prolonged high-intensity intermittent exercise performed in an innovative and ecological form through a Cross-Country mountain biking race leads to central nervous system fatigue. Specifically, HIIE impaired information processing efficiency during a decision-making task and decreased cortical arousal in the late bouts of exercise. There was also an early decrement of cognitive performance (decrease of response accuracy) in the first half of the time-trial race, which can be attributed to the specific nature of HIIE. Interestingly, ingestion of a 7\% carbohydrate-electrolytes solution before and during the TT race is an effective means to postpone central nervous system fatigue. These beneficial effects have been highlighted through maintenance of cortical arousal and sensory efficiency, as well as an enhancement of speed and accuracy of information processing associated with a decrease of perceived exertion during exercise. These findings open new perspectives concerning the use of $\mathrm{CHO}$ in the field of nutritional science and cognitive psychology. Interestingly, coaches and riders in cross-country mountain biking but also in other similar events like mountain bike orienteering or cross-country skiing, may wish to consider using carbohydrate-electrolyte ingestion as an effective nutritional strategy to enhance decision-making performance.

Acknowledgments: The authors wish to thank NHS for providing sports drinks used during the experimental session and Jerome Laurin for the proofreading of the final version of this manuscript. We also extend our gratitude to Caroline Nicol, Dorian Giraud, 
Jason Portal, Denis Bertin, Richard Gamba and Clément Astouric, all of whom assisted with the implementation of experimental protocol and data collection. Last, but not least, we would like to acknowledge all the athletes of the Technoteam 13, who took part in this study, for their time and effort.

Authors contributions: Conceptualization: LP, AH, JB \& KD; Data curation: TG; Formal analysis: LP \& TG; Funding acquisition: LP, JB \& KD; Investigation: LP \& AH; Methodology: LP, AH, JB \& KD; Project administration: LP \& AH; Resources: LP; Software: LP; Supervision: LP; Validation: LP \& TG; Visualization: LP, TG \& KD; Writing - original draft: LP, TG, JB \& KD.

Conflicts of Interest: The authors declare no conflict of interest.

\section{References}

1. Jeukendrup, A. A Step Towards Personalized Sports Nutrition: Carbohydrate Intake During Exercise. Sports Med 2014, 44, 25-33, doi:10.1007/s40279-014-0148-z.

2. Stellingwerff, T.; Cox, G.R. Systematic Review: Carbohydrate Supplementation on Exercise Performance or Capacity of Varying Durations. Appl. Physiol. Nutr. Metab. 2014, 39, 998-1011, doi:10.1139/apnm-2014-0027.

3. Thomas, D.T.; Erdman, K.A.; Burke, L.M. Position of the Academy of Nutrition and Dietetics, Dietitians of Canada, and the American College of Sports Medicine: Nutrition and Athletic Performance. Journal of the Academy of Nutrition and Dietetics 2016, 116, 501-528, doi:10.1016/j.jand.2015.12.006.

4. Baker, L.; Rollo, I.; Stein, K.; Jeukendrup, A. Acute Effects of Carbohydrate Supplementation on Intermittent Sports Performance. Nutrients 2015, 7, 5733-5763, doi:10.3390/nu7075249.

5. Pomportes, L.; Brisswalter, J.; Hays, A.; Davranche, K. Effects of Carbohydrate, Caffeine, and Guarana on Cognitive Performance, Perceived Exertion, and Shooting Performance in High-Level Athletes. International Journal of Sports Physiology and Performance 2019, 14, 576-582, doi:10.1123/ijspp.2017-0865.

6. Collardeau, M.; Brisswalter, J.; Vercruyssen, F.; Audiffren, M.; Goubault, C. Single and Choice Reaction Time during Prolonged Exercise in Trained Subjects: Influence of Carbohydrate Availability. European Journal of Applied Physiology 2001, 86, 150-156, doi:10.1007/s004210100513.

7. Donohoe, R.T.; Benton, D. Cognitive Functioning Is Susceptible to the Level of Blood Glucose. Psychopharmacology 1999, 145, 378-385, doi:10.1007/s002130051071.

8. Mohr, M.; Krustrup, P.; Bangsbo, J. Fatigue in Soccer: A Brief Review. Journal of Sports Sciences 2005, 23, 593-599, doi:10.1080/02640410400021286.

9. McMorris, T.; Hale, B.J.; Corbett, J.; Robertson, K.; Hodgson, C.I. Does Acute Exercise Affect the Performance of Whole-Body, Psychomotor Skills in an Inverted-U Fashion? A Meta-Analytic Investigation. Physiology \& Behavior 2015, 141, 180-189, doi:10.1016/j.physbeh.2015.01.010.

10. De Pauw, K.; Roelands, B.; Knaepen, K.; Polfliet, M.; Stiens, J.; Meeusen, R. Effects of Caffeine and Maltodextrin Mouth Rinsing on P300, Brain Imaging, and Cognitive Performance. Journal of Applied Physiology 2015, 118, 776-782, doi:10.1152/japplphysiol.01050.2014.

11. Chambers, E.S.; Bridge, M.W.; Jones, D.A. Carbohydrate Sensing in the Human Mouth: Effects on Exercise Performance and Brain Activity: Oral Carbohydrate and Exercise Performance. The Journal of Physiology 2009, 587, 1779-1794, doi:10.1113/jphysiol.2008.164285.

12. Welsh, R.S.; Mark Davis, J.; Burke, J.R.; Williams, H.G. Carbohydrates and Physical/Mental Performance during Intermittent Exercise to Fatigue: Medicine \& Science in Sports E Exercise 2002, 34, 723-731, doi:10.1097/00005768-200204000-00025.

13. Stroop, J.R. Studies of Interference in Serial Verbal Reactions. Journal of Experimental Psychology 1935, 18, 643-662, doi:10.1037/h0054651.

14. Dupuy, O.; Tremblay, J. Impact of Carbohydrate Ingestion on Cognitive Flexibility and Cerebral Oxygenation during High-Intensity Intermittent Exercise: A Comparison between Maple Products and Usual Carbohydrate Solutions. Nutrients 2019, 11(9), 2019; doi.org/10.3390/nu11092019

15. Hays, A.; Devys, S.; Bertin, D.; Marquet, L.; Brisswalter, J. Understanding the Physiological Requirements of the Mountain Bike CrossCountry Olympic Race Format. Front. Physiol. 2018, 9, 1062, doi:10.3389/fphys.2018.01062.

16. Hurst, H.T.; Atkins, S. Power Output of Field-Based Downhill Mountain Biking. Journal of Sports Sciences 2006, 24, 1047-1053, doi:10.1080/02640410500431997.

17. Simon, J.R.; Rudell, A.P. Auditory S-R Compatibility: The Effect of an Irrelevant Cue on Information Processing. Journal of Applied Psychology 1967, 51, 300-304, doi:10.1037/h0020586.

18. Lambourne, K.; Audiffren, M.; Tomporowski, P.D. Effects of Acute Exercise on Sensory and Executive Processing Tasks: Medicine $\mathcal{E}$ Science in Sports \& Exercise 2010, 42, 1396-1402, doi:10.1249/MSS.0b013e3181cbee11.

19. Parrott, A. Critical Flicker Fusion Thresholds and Their Relationship to Other Measures of Alertness. Pharmacopsychiatry 1982, 15, 3943, doi:10.1055/s-2007-1019548.

20. Davranche, K.; Pichon, A. Critical Flicker Frequency Threshold Increment after an Exhausting Exercise. Journal of Sport and Exercise Psychology 2005, 27, 515-520, doi:10.1123/jsep.27.4.515.

21. Ueda, T.; Nabetani, T.; Teramoto, K. Differential Perceived Exertion Measured Using a New Visual Analogue Scale during Pedaling and Running. J Physiol Anthropol 2006, 25, 171-177, doi:10.2114/jpa2.25.171.

22. Burke, L.M.; Hawley, J.A.; Wong, S.H.S.; Jeukendrup, A.E. Carbohydrates for Training and Competition. Journal of Sports Sciences 2011, 29, S17-S27, doi:10.1080/02640414.2011.585473. 
23. Gajdos, T.; Servant, M.; Hasbroucq, T.; Davranche, K. A Measure of the Interference Effect Distribution. Behav Res 2020, 52, 16291639, doi:10.3758/s13428-019-01339-6.

24. Bates, D.; Mächler, M.; Bolker, B.; Walker, S. Fitting Linear Mixed-Effects Models Using Lme4. arXiv:1406.5823 [stat] 2014.

25. Kuznetsova, A.; Brockhoff, P.B.; Christensen, R.H.B. LmerTest Package: Tests in Linear Mixed Effects Models. J. Stat. Soft. 2017, 82, doi:10.18637/jss.v082.i13.

26. Presland, J.D.; Dowson, M.N.; Cairns, S.P. Changes of Motor Drive, Cortical Arousal and Perceived Exertion Following Prolonged Cycling to Exhaustion. Eur J Appl Physiol 2005, 95, 42-51, doi:10.1007/s00421-005-1395-3.

27. Grego, F.; Vallier, J.-M.; Collardeau, M.; Rousseu, C.; Cremieux, J.; Brisswalter, J. Influence of Exercise Duration and Hydration Status on Cognitive Function During Prolonged Cycling Exercise. Int J Sports Med 2005, 26, 27-33, doi:10.1055/s-2004-817915.

28. Backhouse, S.H.; Ali, A.; Biddle, S.J.H.; Williams, C. Carbohydrate Ingestion during Prolonged High-Intensity Intermittent Exercise: Impact on Affect and Perceived Exertion: CHO Ingestion Improves Perceived Activation. Scandinavian Journal of Medicine $\mathcal{E}$ Science in Sports 2007, 17, 605-610, doi:10.1111/j.1600-0838.2006.00613.x.

29. Tomporowski, P.D. Effects of Acute Bouts of Exercise on Cognition. Acta Psychologica 2003, 112, 297-324, doi:10.1016/S00016918(02)00134-8.

30. Eddy, M.D.; Hasselquist, L.; Giles, G.; Hayes, J.F.; Howe, J.; Rourke, J.; Coyne, M.; O’Donovan, M.; Batty, J.; Brunyé, T.T.; et al. The Effects of Load Carriage and Physical Fatigue on Cognitive Performance. PLoS ONE 2015, 10, e0130817, doi:10.1371/journal.pone.0130817.

31. Bottoms, L.M.; Hunter, A.M.; Galloway, S.D.R. Effects of Carbohydrate Ingestion on Skill Maintenance in Squash Players. European Journal of Sport Science 2006, 6(3), 187-195,doi: 10.1080/17461390600804455

32. Turner, C.E.; Byblow, W.D.; Stinear, C.M.; Gant, N. Carbohydrate in the Mouth Enhances Activation of Brain Circuitry Involved in Motor Performance and Sensory Perception. Appetite 2014, 80, 212-219, doi:10.1016/j.appet.2014.05.020.

33. Miller, M.G.; Hanson, N.; Tennyck, J.; Plantz, K. A Comparison of High-Intensity Interval Training (HIIT) Volumes on Cognitive Performance. J Cogn Enhanc 2019, 3, 168-173, doi:10.1007/s41465-018-0107-y.

34. Tsukamoto, H.; Suga, T.; Takenaka, S.; Tanaka, D.; Takeuchi, T.; Hamaoka, T.; Isaka, T.; Ogoh, S.; Hashimoto, T. Repeated HighIntensity Interval Exercise Shortens the Positive Effect on Executive Function during Post-Exercise Recovery in Healthy Young Males. Physiology \& Behavior 2016, 160, 26-34, doi:10.1016/j.physbeh.2016.03.029.

35. Davranche, K.; Brisswalter, J.; Radel, R. Where Are the Limits of the Effects of Exercise Intensity on Cognitive Control? Journal of Sport and Health Science 2015, 4, 56-63, doi:10.1016/j.jshs.2014.08.004.

36. Chang, Y.K.; Labban, J.D.; Gapin, J.I.; Etnier, J.L. The Effects of Acute Exercise on Cognitive Performance: A Meta-Analysis. Brain research 2012,1453, 87-101.doi: 10.1016/j.brainres.2012.02.068.

37. Bischoff, J. The Effects of Blood Glucose Levels on Cognitive Performance: A Review of the Literature. $2007,142$.

38. Felig, P.; Cherif, A.; Minagawa, A.; Wahren, J. Hypoglycemia during Prolonged Exercise in Normal Men. N Engl J Med 1982, 306, 895-900, doi:10.1056/NEJM198204153061503.

39. Rollo, I.; Homewood, G.; Williams, C.; Carter, J.; Goosey-Tolfrey, V.L. The Influence of Carbohydrate Mouth Rinse on Self-Selected Intermittent Running Performance. International Journal of Sport Nutrition and Exercise Metabolism 2015, 25, 550-558, doi:10.1123/ijsnem.2015-0001.

40. Noble B J; Robertson R J Perceived Exertion. In; Human Kinetics Champaign, 1996.

41. Bendiksen, M.; Bischoff, R.; Randers, M.B.; Mohr, M.; Rollo, I.; Suetta, C.; Bangsbo, J.; Krustrup, P. The Copenhagen Soccer Test: Physiological Response and Fatigue Development. Medicine $\mathcal{E}$ Science in Sports $\mathcal{E}$ Exercise 2012, 44, 1595-1603, doi:10.1249/MSS.0b013e31824cc23b.

42. Nicholas, C.W.; Tsintzas, K.; Boobis, L.; Williams, C. Carbohydrate-Electrolyte Ingestion during Intermittent High-Intensity Running: Medicine \& Science in Sports \& Exercise 1999, 31, 1280-1286, doi:10.1097/00005768-199909000-00008.

43. Bjorkman, O.; Sahlin, K.; Hagenfeldt, L.; Wahren, J. Influence of Glucose and Fructose Ingestion on the Capacity for Long-Term Exercise in Well-Trained Men. Clin Physiol 1984, 4, 483-494, doi:10.1111/j.1475-097X.1984.tb00134.x. 